\title{
Estudo por Imagem da Artrite Reumatóide no Quadril(*)
}

\author{
Hip Imasing in Rheumatoid Arthritis
}

\author{
Wanda Chiyoko Iwakami Caldana ${ }^{(1)}$, Rozana Mesquita Ciconelli ${ }^{(2)}$, Artur da Rocha Correa Fernandes ${ }^{(3)}$
}

\section{RESUMO}

Este artigo de revisão tem como objetivo discutir os principais aspectos por imagem do envolvimento do quadril em adultos pela artrite reumatóide, com abordagem ilustrativa dos principais achados. Os métodos de imagem estudados são a radiografia, ultra-sonografia, tomografia computadorizada e a ressonância magnética com o uso do contraste paramagnético (gadolínio) pela via intravenosa.

Palavras-chave: artrite reumatóide, quadril, imagem, ressonância magnética, gadolínio.

O diagnóstico da artrite reumatóide (AR) no quadril pode representar um desafio para o radiologista, principalmente ao considerarmos a menor freqüência de envolvimento dessa articulação pela doença. Além disso, não é rara a sobreposição de achados com outras alterações articulares, com destaque para os processos degenerativos ${ }^{(1)}$. Por esses motivos, é de fundamental importância o completo domínio dos achados indicativos da AR, a fim de orientar a suspeita diagnóstica na direção mais adequada. Sendo assim, elaboramos esta revisão com o objetivo de discutir os principais aspectos por imagem do envolvimento do quadril pela AR, com abordagem ilustrativa dos principais achados.

\section{ASPECTOS FISIOPATOLÓGICOS DA ARTRITE REUMATÓIDE}

Na patogênese da AR, acredita-se que um evento desencadeante imune-mediado possa ser o gatilho para o desenvolvimento do complexo fisiopatológico da doença.

\begin{abstract}
The aim of this article is to discuss the main aspects of the hip involvement in adults with rheumatoid arthritis. The diagnostic imaging methods that will be illustrated are radiography, ultrasonography, computer tomography and magnetic resonance imaging (MRI) with gadolinium endovenous injection.
\end{abstract}

Keywords: rheumatoid arthritis, hip, imaging, magnetic resonance imaging, gadolinium.

Especula-se que esse evento, ainda pouco conhecido, poderia corresponder à infecção por algum patógeno desconhecido, ou agentes como retrovírus, parvovírus, vírus de Epstein-Barr ou fungos como a cândida ${ }^{(2)}$.

Fatores genéticos sugeridos pela maior freqüência da doença em portadores do antígeno linfocitário HLA-DR4 seriam responsáveis pela predisposição individual no desenvolvimento da resposta inflamatória crônica sistêmica, de predominante envolvimento articular, que caracteriza a doença ${ }^{(2,3)}$.

Iniciado o processo imunológico, uma série de efeitos em cascata é desencadeada na articulação, começando com a ativação de linfócitos $\mathrm{T}$, produção de interleucinas e estímulo às células sinoviais para a produção de proteinases, que causam a agressão à matriz cartilaginosa. Há também estímulo à produção de imunoglobulinas (fator reumatóide), com a formação de imunocomplexos que se depositam na membrana sinovial, ativando o sistema complemento e promovendo a progressiva migração de neutrófilos e macrófagos para o local ${ }^{(4)}$.

\footnotetext{
* Trabalho realizado no Departamento de Diagnóstico por Imagem (DDI) e na Disciplina de Reumatologia da Universidade Federal de São Paulo (Unifesp-EPM), São Paulo, SP, Brasil. Recebido em 22/07/2004. Aprovado, após revisão, em 09/02/2005.

1. Pós-graduanda do Departamento de Diagnóstico por Imagem da Unifesp-EPM.

2. Docente da Disciplina de Reumatologia da Unifesp-EPM.

3. Docente do Departamento de Diagnóstico por Imagem da Unifesp-EPM.

Endereço para correspondência: Dra. Wanda Chiyoko Iwakami Caldana. Rua Joaquim Távora, 1299 - apto. 82, CEP 04015 002. São Paulo, SP, Brasil. Tel./fax 55 (1 1) 5084-3551; e-mail: wiwakami@ig.com.br
} 
Esse processo imunológico resultará em proliferação e espessamento sinovial, com áreas de necrose fibrinóide. À necrose, segue-se uma tentativa de reparação com angiogênese sinovial e a formação de um tecido de granulação hipertrófico rico em macrófagos, conhecido como pannus. Nessa fase, o quadro clínico geralmente manifesta-se como edema, aumento da temperatura local e dor articular (características inflamatórias) ${ }^{(5,6)}$.

Às alterações inflamatórias articulares sobrepõe-se a progressiva desmineralização do osso subcondral (osteopenia periarticular). A seguir, a proliferação do pannus determina erosões na cartilagem hialina de revestimento, com exposição cortical, erosões ósseas inicialmente marginais, e progressiva destruição arquitetural, com perda do alinhamento e congruência articular. O estágio mais avançado da doença caracteriza-se pelo predomínio da fibrose, resultado final do tecido de granulação destrutivo, com anquilose, deformidades e reabsorção óssea ${ }^{(4)}$.

\section{EXPRESSÃO RADIOGRÁFICA DAS ALTERAÇÕES}

As alterações radiográficas da AR são bem correlacionadas com os achados patológicos descritos por Resnick ${ }^{(7)}$. Um dos primeiros sinais radiográficos da doença é o aumento de partes moles periarticulares de forma fusiforme e simétrica, refletindo a sinovite, congestão e edema da membrana sinovial com acúmulo de eritrócitos, linfócitos e células polimorfonucleares, associado à distensão líquida capsular. Em resposta ao processo inflamatório, surge a osteopenia periarticular. Com a progressão da doença, a proliferação sinovial que destrói a superfície condral promove redução do espaço articular e erosões ósseas subcondrais marginais e centrais. Em estágios mais avançados, subluxações, deformidades e anquilose óssea podem ser vistas na radiografia, representando a perda da função $\operatorname{articular}^{(8-9)}$.

\section{O QUADRIL NA ARTRITE REUMATÓIDE}

O envolvimento das grandes articulações pela AR é em geral bastante extenso, com predominante acometimento dos membros superiores e joelhos. O quadril e o tornozelo são articulações menos freqüentemente envolvidas ${ }^{(8,10)}$, cujo comprometimento parece aumentar com a duração e a gravidade da doença, principalmente nos pacientes com uso prolongado de corticosteróides ${ }^{(8)}$.

A prevalência da AR no quadril é um dado controverso na literatura, em função da heterogeneidade de parâmetros utilizados para seu diagnóstico em trabalhos de metodologia não comparável (prospectivos e retrospectivos), que nem sempre consideram o tempo de evolução da doença. Para ilustrar esse aspecto, é interessante destacar alguns trabalhos que enfocam alterações radiográficas nas grandes articulações. Em 1963, Glick et al., revisando trabalhos de vários autores, estimaram a freqüência da AR no quadril em torno de $10 \%$ a 33\% ${ }^{(11)}$. Em 1969, Duthie et al. relataram uma prevalência em torno de $40 \%$, alta, porém atribuída a pacientes mais graves e hospitalizados ${ }^{(12)}$. Já um estudo prospectivo de oito anos realizado na década de 1970 mostrou um envolvimento do quadril em 13\% dos casos, com predomínio de alterações ósseas de grau moderado ${ }^{(13)}$. De forma semelhante, em 1995, Eberhardt realizou um estudo prospectivo em portadores de AR, acompanhando sua evolução por cinco anos e constatou que 15\% dos pacientes tinham envolvimento do quadril no período inicial da doença (um ano), com piora progressiva da função articular e destruição articular em $28 \%$ dos pacientes ${ }^{(10,14)}$. Já Roberts et al. encontraram, em 1988, alterações no quadril em 26\% dos pacientes com cinco anos de atividade da doença ${ }^{(15)}$. Outros estudos retrospectivos apontam prevalência entre $10 \%$ e 17\% em pacientes com tempo de doença inferior a dez anos, enquanto aqueles com maior tempo de evolução entre $36 \%$ e $40 \%$ apresentam doença no quadril ${ }^{(12,16,17)}$.

Todos os estudos publicados indicam que o quadril pode ser acometido pela AR desde o início da doença, com a destruição progressiva da articulação relacionada principalmente ao tempo de evolução ${ }^{(18)}$. Há também indícios de piora do prognóstico quando a artropatia se instala no quadril já no período inicial da doença ${ }^{(19)}$.

$\mathrm{O}$ quadril tem algumas particularidades que podem dificultar a detecção precoce da AR, bem como o monitoramento da progressão ou remissão da doença: a anatomia profunda não permite caracterizar derrame ou sinovite pelo aumento do volume ou temperatura local; por ser uma articulação de sustentação do peso, pode sofrer alterações biomecânicas e causar osteoartrose, ocultando a artropatia inflamatória eventualmente associada.

Outro aspecto de relevância está na sintomatologia do envolvimento articular do quadril. Entre os sintomas descritos, os pacientes com AR podem apresentar dor local ou irradiada, alteração da marcha por disfunção biomecânica decorrente de tendinopatia dos glúteos, encurtamento do membro acometido e dificuldade ou mesmo limitação para alguns movimentos como a rotação interna e externa, flexão, extensão, adução e abdução ${ }^{(8)}$. $\mathrm{O}$ exame clínico pode ainda demonstrar aumento de partes moles na região 
anterior do quadril ou do trocânter maior na vigência de bursites do iliopsoas e trocantérica, respectivamente ${ }^{(20,21)}$. $\mathrm{Na}$ evolução crônica, o envolvimento pode estender-se para os grupamentos musculares causando atrofia por desuso, formação de nódulos reumatóides sobre o sacro e tuberosidades isquiáticas, além de edema dos membros inferiores decorrentes da compressão vascular por cistos sinoviais $^{(8)}$.

Sabe-se que alguns pacientes com AR podem estar assintomáticos mesmo na vigência de sinovite, dificultando ainda mais a sua detecção, já que o estudo por imagem do quadril geralmente não é realizado na ausência de queixa local ${ }^{(14,16,18)}$.

A osteonecrose não é incomum nos pacientes com AR e pode ocorrer como complicação do uso prolongado de corticosteróides $^{(8,22)}$. Não se sabe, contudo, a real prevalência da osteonecrose em pacientes submetidos à corticoterapia, a relação com o tempo de uso e a dose empregada. Os achados na radiografia são tardios e muitas vezes torna-se difícil sua diferenciação com a osteoartrose, por apresentar alterações degenerativas semelhantes ${ }^{(22)}$. Há evidências de que a osteonecrose pode não ser conseqüência do uso prolongado ou de altas doses do corticosteróide, mas da própria fisiopatogenia da AR, que evolui para osteoporose e inflamação intra-articular, pelo uso de outros medicamentos como antiinflamatórios não hormonais, e até por alterações na estrutura vascular da cabeça femoral presente em alguns indivíduos, o que predispõe à isquemia sangüínea local(22,23). Uma extensa revisão sobre o uso de corticosteróides na AR mostrou que a administração intra-articular pode predispor à osteonecrose quando utilizada em altas doses ou em intervalos curtos ${ }^{(24)}$.

\section{DIAGNÓSTICO DIFERENCIAL}

O quadril pode ser envolvido por outras doenças mesmo em pacientes portadores de AR. Entre os principais achados patológicos, destacam-se a osteoartrose ${ }^{(7)}$, fratura por insuficiência, o edema transitório do quadril ${ }^{(21)}$, artrite séptica e tendinopatias dos músculos abdutores da coxa que se inserem no trocânter maior ${ }^{(25-27)}$.

Por fim, existe ainda um grupo de alterações caracterizadas por dor referida no quadril, sem substrato anatômico de lesão articular. Nesses casos, é preciso considerar a coluna vertebral que apresenta focos nociceptivos das raízes nervosas, das facetas articulares e ligamentos paraespinhosos da coluna lombar que podem promover dor referida na região lateral do quadril ${ }^{(28)}$.

\section{MÉTODOS DE ESTUDO POR IMAGEM DO QUADRIL NA ARTRITE REUMATÓIDE}

\section{RADIOGRAFIA}

Poucos são os trabalhos publicados que abordam o comprometimento do quadril pela AR. Em sua maioria, o substrato de estudo desses trabalhos consiste na avaliação das alterações radiográficas da doença ${ }^{(11,12,16)}$. Nesse sentido, a radiografia tem papel bem estabelecido quanto à caracterização da evolução da doença ${ }^{(3)}$, por ser de baixo custo, ter ampla disponibilidade e ser de fácil execução ${ }^{(29,30)}$. O quadril pode ser avaliado obtendo-se incidências radiográficas de frente, (ântero-posterior) e obliquas em abdução (posição de rã).

Por outro lado, a radiografia tem baixa sensibilidade para as alterações iniciais da doença, sendo também inadequada para monitorar a eficácia do tratamento ${ }^{(3,31)}$.

Ao contrário do que ocorre nas articulações superficiais, no quadril o aumento de partes moles não corresponde a um achado habitual da AR, principalmente pela sua localização profunda. De acordo com a literatura, o primeiro sinal radiográfico da doença no quadril é a perda simétrica e bilateral do espaço articular, seguida de esclerose subcondral circundada por osteopenia, resultado do afilamento da cartilagem que recobre a cabeça femoral e o acetábulo pela invasão do pannus sinovial ${ }^{(7)}$. Erosões marginais, tão freqüentes no comprometimento das outras articulações, têm pouca expressão no quadril ${ }^{(12,32)}$.

As articulações de carga afetadas pela AR em razão da redistribuição do peso sustentado são mais susceptíveis ao processo degenerativo secundário. Nos casos mais graves, em que a deformidade articular resulta em alteração significativa da biomecânica, podem ser encontrados sinais radiográficos de doença degenerativa secundária ao processo inflamatório inicial. Esses achados são caracterizados por formação de cistos subcondrais, esclerose cortical, osteofitose marginal e até mesmo colapso do acetábulo e da cabeça femoral, refletindo um processo de reparação decorrente da destruição óssea e cartilaginosa ${ }^{(8,12)}$ (Figura 1).

Nos casos avançados, pode ocorrer a migração axial da cabeça femoral em direção ao acetábulo, efeito conhecido como protrusão acetabular, decorrente da tração muscular sobre as estruturas ósseas fragilizadas, resultando na migração progressiva da cabeça femoral induzida pela remodelação do acetábulo ${ }^{(8,21,33)}$.

A radiografia consegue diferenciar outras alterações do quadril, como a osteoartrose e a osteonecrose da cabeça. Isto só é possível, no entanto, nas fases tardias, após a instalação das lesões ${ }^{(34)}$. 


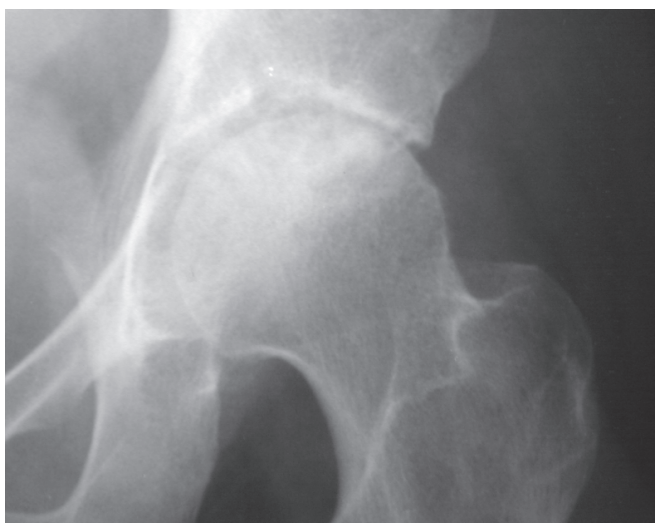

FiguRA 1 - Radiografia simples do quadril: redução do espaço articular superior com erosões, cistos e esclerose subcondrais na cabeça femoral. 0 acetábulo apresenta irregularidade e esclerose subcondral. Há também esboço de osteófitos marginais

\section{ULTRA-SONOGRAFIA}

A ultra-sonografia tem as vantagens de ser um método rápido, não invasivo, amplamente disponível e de baixo custo. Sua capacidade diagnóstica envolve as principais alterações de partes moles, podendo evidenciar bursites, tendinopatias, espessamentos sinoviais, sinais de sinovite, derrame articular, ou alterações vasculares (Figuras 2 e 3). Em alguns casos, pode demonstrar até mesmo afilamento e erosões condrais, ou presença de corpos livres intra-articulares ${ }^{(31,35-37)}$. Além dos procedimentos diagnósticos, a ultra-sonografia pode também ser útil em procedimentos invasivos como na orientação de punções aspirativas ${ }^{(31,35-37)}$.

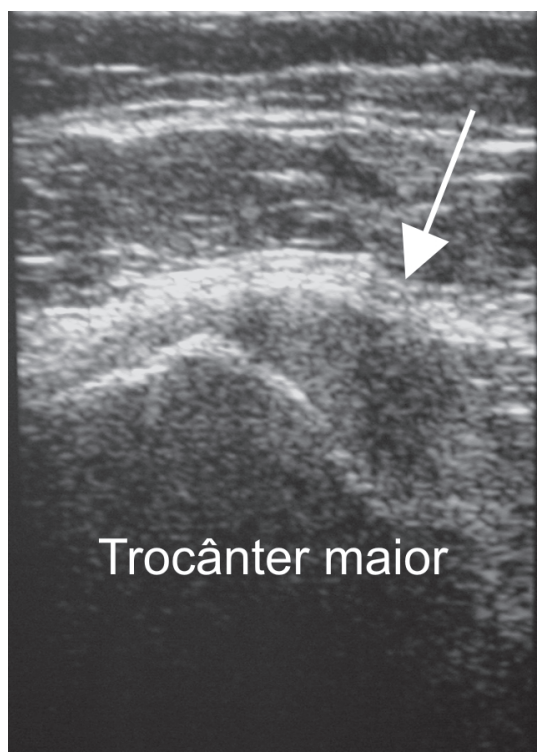

FIGURA 2 - Ultra-sonografia mostrando bursite trocantérica (seta)

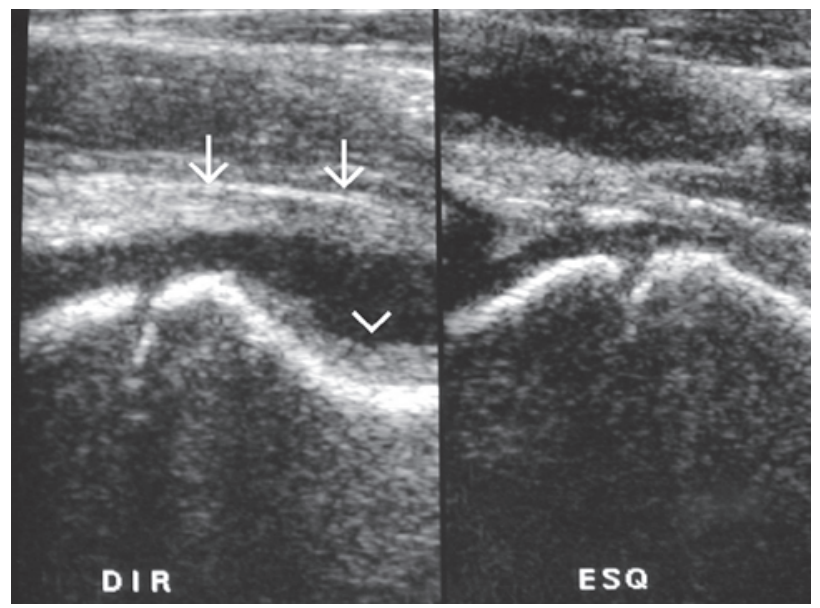

FiguRa 3 - Ultra-sonografia do quadril no nível da cabeça/colo femoral mostrando derrame (cabeça de seta) e espessamento sinovial no quadril direito (setas)

De maneira ideal, o exame ultra-sonográfico deve ser realizado com transdutor linear de alta frequiência ( 7 a 15 $\mathrm{MHz})$, capaz de gerar imagens com melhor resolução espacial e detalhamento anatômico ${ }^{(4)}$.

$\mathrm{Na}$ AR, a ultra-sonografia destaca-se pela facilidade na detecção de sinovite tanto em pacientes sintomáticos como naqueles sem sintomatologia inflamatória ${ }^{(14)}$. É particularmente útil na artrite idiopática juvenil (AIJ), quando há maior incidência de acometimento do quadril. Nesses pacientes, a ultra-sonografia pode reduzir a morbidade da AIJ, por detectar precocemente as alterações, oferecendo um método útil no controle terapêutico e acompanhamento evolutivo ${ }^{(31,36)}$.

Outro recurso disponível no estudo ultra-sonográfico é a associação com o Doppler colorido, permitindo em casos de espessamento da membrana sinovial, a caracterização de sinovite. Isto pode ser feito pela demonstração do aumento da vascularização sinovial ${ }^{(4,38,39)}$. No entanto, o acesso para estudo articular por meio da ultra-sonografia é restrito em comparação a outros métodos, em razão da limitação determinada pela não-penetração do feixe acústico em estruturas ósseas, impossibilitando a avaliação de toda a cavidade articular ${ }^{(4,39)}$.

\section{TOMOGRAFIA COMPUTADORIZADA}

A tomografia computadorizada (TC) é a modalidade de escolha para estudar o arcabouço ósseo do quadril ${ }^{(40)}$. Com o advento da TC helicoidal, as reconstruções multiplanares permitiram visualizar alterações decorrentes de traumas, 
osteonecrose, displasias congênitas e como avaliação préoperatória em artroplastias. $\mathrm{Na} \mathrm{AR}$, esse método de imagem é particularmente útil na avaliação de articulações complexas como a transição crânio-vertebral, a articulação têmporo-mandibular, sacro-ilíacas e calcâneo, cuja anatomia é de difícil visualização numa radiografia simples ${ }^{(4,9,41)}$. Assim, erosões corticais, subluxações atlantoaxiais na coluna cervical e irregularidades na superfície articular do côndilo mandibular são bem demonstráveis pela TC, graças à alta resolução espacial do método ${ }^{(9,42)}$. A TC, no entanto, é limitada ao estudo das partes moles periarticulares, particularmente de alterações sinoviais, pois não apresenta resolução de contraste suficiente para caracterizálas ${ }^{(41,43)}$. Assim, o uso da TC do quadril em pacientes com AR tem papel secundário, podendo ser mais bem utilizada na avaliação das erosões e deformidades ósseas, decorrentes da gravidade da doença ${ }^{(41,43)}$. Já a artrografia por TC (artroTC) pode ser útil na avaliação da formação do pannus, na integridade da cartilagem e do lábio acetabular ${ }^{(44)}$.

Recentemente, para o estudo por imagem dos ossos carpais, o grupo da National Institute of Arthritis and Musculoskeletal and Skin Diseases (NIAMS) considerou a TC como método padrão para detecção de erosões ósseas por meio da reconstrução tridimensional, sendo superior à RM nesse aspecto ${ }^{(45)}$.

\section{RESSONÂNCIA MAGNÉTICA}

Apesar de seu custo, a ressonância magnética (RM) constitui o melhor método de imagem para avaliação articu$\operatorname{lar}^{(3,4,30,39,46-50)}$. Entre suas principais vantagens, destacam-se a não invasividade, ausência de radiação ionizante, não utilização do contraste iodado (potencialmente nefrotóxico e alérgeno), e capacidade de melhor detalhamento anatômico, tanto pela natureza multiplanar de aquisição, como pelo elevado contraste entre os diferentes tecidos corporais $^{(3,4,30,39,46,50-53)}$ (Figura 4).

$\mathrm{Na}$ avaliação de pacientes com AR, a RM tem papel bem estabelecido na detecção da atividade inflamatória, caracterizada pela presença de sinovite, muitas vezes sutil ao exame clínico ${ }^{(53)}$. A RM permite também mensurar a extensão do acometimento articular e extra-articular e avaliar as complicações decorrentes do tempo da doença ${ }^{(41)}$. Neste sentido, tem alta sensibilidade para a avaliação de lesões tendíneas e ligamentares, envolvimento da bainha tendínea (tenossinovite), bolsa trocantérica ${ }^{(20)}$, lesões ósseas (erosões subcondrais, cistos) que inicialmente podem não ser vistas pela radiografia convencional, alterações na medular óssea, lesões condrais e na diferenciação entre der- rame articular e sinovite, neste caso por meio do contraste endovenoso (gadolínio) $)^{(3,39-41,45,46,54,55)}$ (Figura 5 a 8).

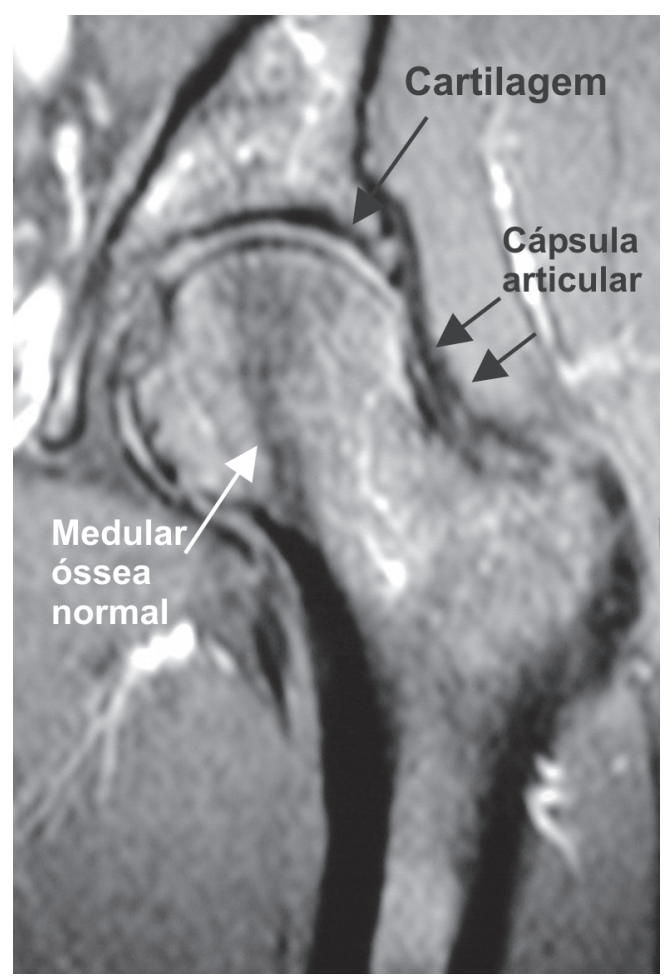

FIgURA 4 - Exame de RM normal do quadril no plano coronal TSE ponderada em T1 após o contraste

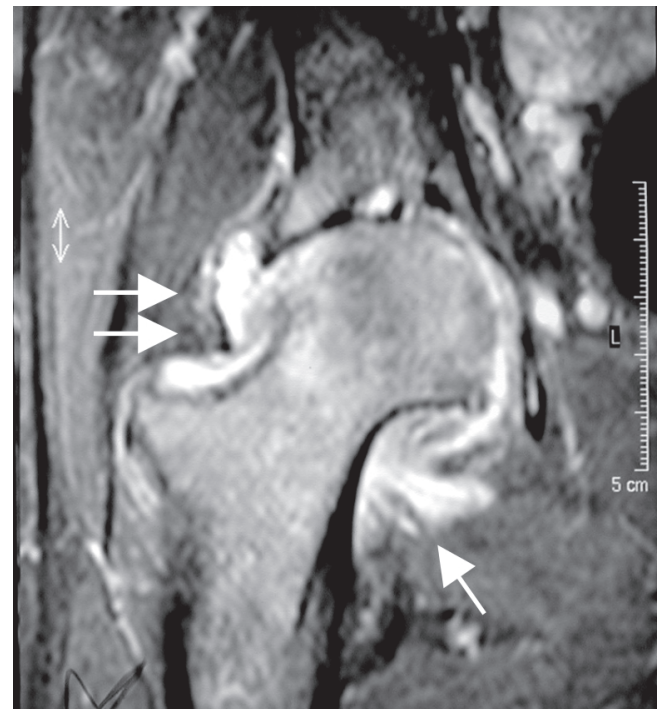

FiguRA 5 - RM do quadril no plano coronal após a injeção endovenosa do contraste: sinovite (setas brancas), edema da medular óssea da cabeça e colo femoral caracterizado por sinal elevado (hipersinal) 

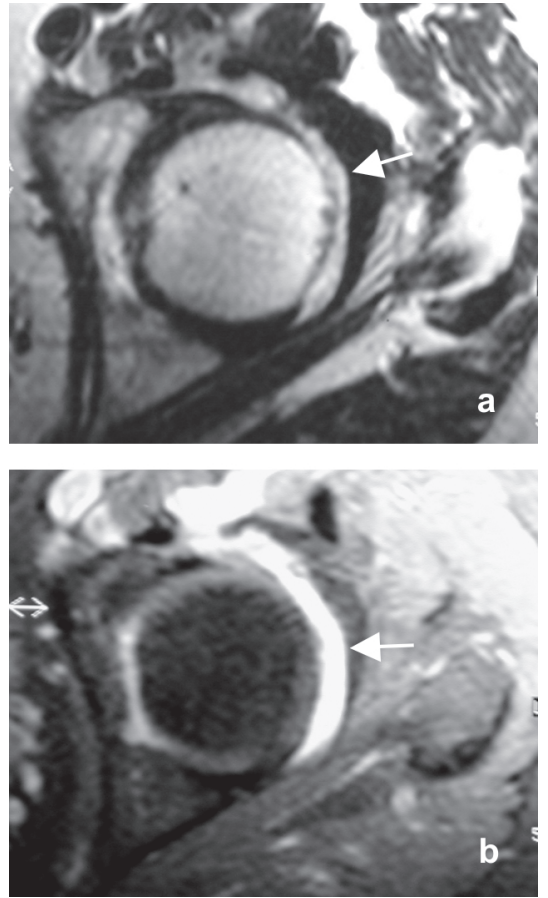

FiguRA 6 - RM do quadril nos planos transversais ponderadas em T2 (a) e após o contraste(b): espessamento sinovial e realce (setas)
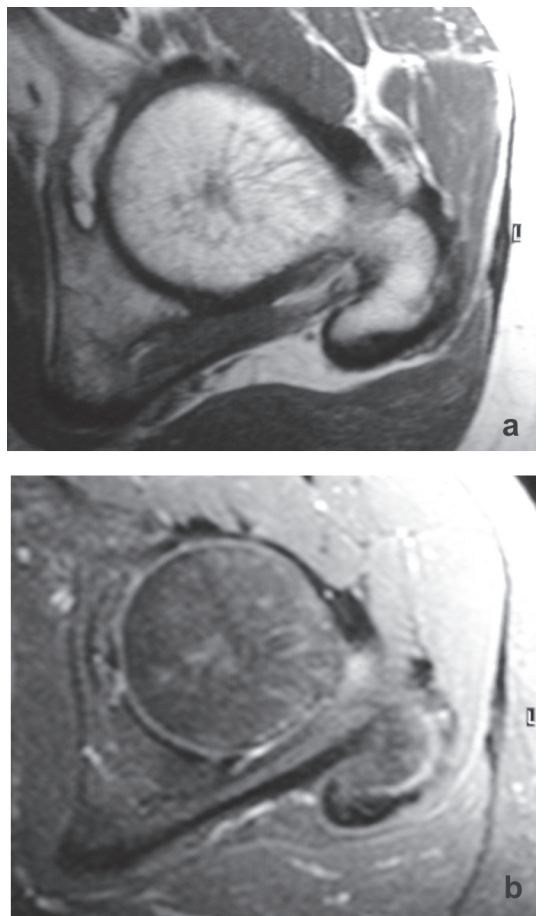

FiguRA 7 - RM do quadril nos planos transversais, ponderadas em T2 (a) e após o contraste(b): exame normal sem sinovite

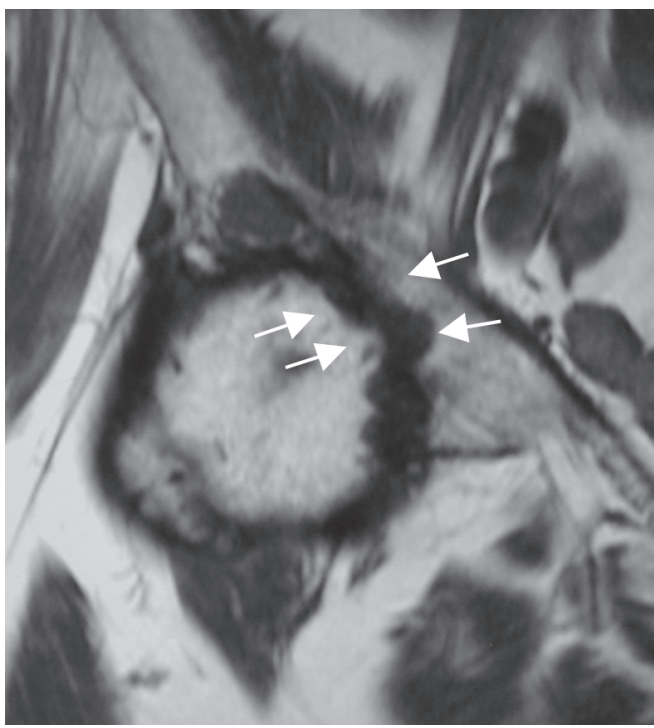

FiguRa 8 - RM do quadril no plano coronal, ponderada em T1: erosões subcondrais na cabeça femoral e no acetábulo

Além das vantagens acima expostas, o estudo do quadril pela RM é particularmente útil na abordagem da integridade da cabeça femoral, podendo detectar alterações iniciais da osteonecrose $^{(23,37)}$, e no diagnóstico diferencial com outras desordens ${ }^{(28)}$.

Em casos selecionados, em que o objetivo principal do exame está na avaliação da cartilagem e lábio acetabular, a injeção intra-articular do contraste paramagnético, denominada artrografia por ressonância magnética (artro-RM), pode aumentar a sensibilidade na detecção de lesões nessas estruturas $^{(48,56,57)}$.

\section{REALCE DA MEMBRANA SINOVIAL AO CONTRASTE (GADOLÍNIO)}

Uma questão técnica deve ser observada na avaliação do realce sinovial na RM. O tempo para a aquisição das imagens após a injeção do meio de contraste é fundamental para a caracterização da sinovite e diferenciação com o derrame articular ${ }^{(58-60)}$. Em indivíduos normais, a membrana sinovial não apresenta realce significativo após a injeção endovenosa do contraste (gadolínio) ${ }^{(6,50,59,61)}$. Nos pacientes com AR, o realce da membrana sinovial é provocado pelo aumento da perfusão e permeabilidade vascular, decorrentes do processo inflamatório; por meio de difusão, o contraste do espaço extracelular da sinóvia difunde-se progressivamente para o líquido articular ${ }^{(6,50,61)}$. Portanto, a aquisição das imagens não deve ultrapassar dez minutos após a injeção endovenosa, pois a partir desse momento o realce sinovial pode 
ser menos evidente pelo início da contrastação do líquido $\operatorname{articular}^{(6,50,53,60-62)}$.

A importância na detecção da sinovite pela RM está na possibilidade de monitorar a evolução do paciente e também avaliar a eficácia dos diferentes métodos terapêuticos ${ }^{(3,6,39-41,46,50,51,54,55,63,64)}$. Podemos mensurar esse processo inflamatório quantificando o volume da membrana sinovial hipertrofiada de forma manual ou semi-automatizada ${ }^{(50,63}$ ${ }^{65-67)}$, ou utilizando estudo dinâmico com contraste endovenoso para calcular a porcentagem e a taxa máxima do pico de realce numa área pré-determinada de uma articulação ${ }^{(50,58,65,67)}$. O estudo dinâmico permite não só caracterizar a sinovite, mas também diferenciá-la entre processo inflamatório agudo hipervascular e o pannus fibroso ${ }^{(58)}$. Já o estudo convencional de RM não fornece tais informações, porém possibilita uma visualização global da articulação caracterizando o espessamento e o realce da membrana sinovial nas aquisições estáticas após o contraste ${ }^{(68)}$.

\section{PERSPECTIVAS FUTURAS}

$\mathrm{Na}$ literatura atual, o principal foco de pesquisa na investigação da AR pela RM contrastada está no estabelecimento de critérios diagnósticos para esse método, a exemplo do que já existe pela radiografia ${ }^{(45)}$.

Recentemente, muitos autores vêm estudando a utilidade da RM na detecção precoce de lesões ósseas e sinovite nas mãos, previamente às alterações radiográficas, no intuito de quantificar os achados por meio de escalas e graduações, já existentes pela radiografia (Larsen, Sharp) ${ }^{(55,69)}$. Para tornar a RM um método confiável e aplicável como a radiografia na caracterização da AR e na sua evolução, é necessário padronizar as aquisições das seqüências, determinar o tipo e o local das lesões a serem graduadas e criar uma nomenclatura para tornar possível a utilização desse método por

\section{REFERÊNCIAS}

1. Caldana W: A ressonância magnética do quadril na avaliação de pacientes com artrite reumatóide - estudo descritivo [Mestrado]. São Paulo, Universidade Federal de São Paulo, 2004.

2. Burmester GR: Hit and run or permanent hit? Is there evidence for a microbiological cause of rheumatoid arthritis? J Rheumatol 18 1443-7, 1991.

3. Winalski CS, Palmer WE, Rosenthal DI, Weissman BN: Magnetic resonance imaging of rheumatoid arthritis. Radiol Clin North Am 34: 243-58, x, 1996.

4. Imhof H, Nobauer-Huhmann IM, Gahleitner A, et al: Pathophysiology and imaging in inflammatory and blastomatous synovial diseases. Skeletal Radiol 31: 313-33, 2002. qualquer grupo científico ${ }^{(45)}$. Em 2001, realizou-se um encontro internacional da subcomissão do ACR OMERACT (American College of Rheumatology Outcome Measures in Rheumatoid Arthritis Clinical Trials), quando se propôs uma padronização com relação à técnica de RM utilizada nas mãos e punhos, quanto à graduação das erosões ósseas, edema da medular óssea e sinovite ${ }^{(69)}$. Assim, um grupo iniciou um estudo multicêntrico graduando a sinovite, lesões ósseas (erosões) e redução do espaço articular nos dedos (articulações metacarpofalângicas) e punhos, encontrando uma relativa consistência na interpretação dessas alterações entre os observadores participantes de vários países $^{(70)}$. Recentemente, o mesmo grupo publicou resultados parciais de um estudo prospectivo de quatro anos, determinando a sinovite, edema da medular óssea e lesões ósseas como indicadores da atividade da doença ${ }^{(55)}$.

\section{CONCLUSÃO}

A avaliação do quadril na AR é um desafio diagnóstico tanto para reumatologistas quanto para radiologistas, por ser essa uma articulação profunda que não permite adequada avaliação física e por ser uma área de sobrecarga mecânica, propiciando alterações degenerativas secundárias ou não à AR. Portanto, os métodos de imagem são importantes como ferramentas complementares na avaliação articular dos pacientes. A radiografia simples tem boa utilidade como abordagem propedêutica inicial, porém a complementação com métodos mais sofisticados como a tomografia ou a ressonância pode ser fundamental na avaliação dos casos mais complexos, em que há possibilidade de associação com outros processos patológicos. Para essa finalidade, a RM tem papel de destaque por permitir a avaliação da medular óssea, estruturas intra-articulares e partes moles periarticulares e possibilitar a caracterização da sinovite.

5. Harris ED Jr: Rheumatoid arthritis. Pathophysiology and implications for therapy. N Engl J Med 322: 1277-89, 1990.

6. Kursunoglu-Brahme S, Riccio T, Weisman MH, et al: Rheumatoid knee: role of gadopentetate-enhanced MR imaging. Radiology 176: 831-5, 1990.

8. Resnick D, Niwayama G: Diagnosis of bone and joint disorders. 2nd ed. Philadelphia: Saunders, 1988.

7. Resnick D: Common disorders of synovium-lined joints: pathogenesis, imaging abnormalities, and complications. AJR Am J Roentgenol 151: 1079-93, 1988.

9. Scutellari PN, Orzincolo C: Rheumatoid arthritis: sequences. Eur J Radiol 27 Suppl 1: S31-8, 1988.

10. Drossaers-Bakker KW, Kroon HM, Zwinderman AH, Breedveld FC, Hazes JM: Radiographic damage of large joints in long-term 
rheumatoid arthritis and its relation to function. Rheumatology (Oxford) 39: 998-1003, 2000.

11. Glick E, Mason R, Wenley W: Rheumatoid Arthritis Affecting The Hip Joint. Ann Rheum Dis 22: 416-23, 1963.

12. Duthie RB, Harris CM: A radiographic and clinical survey of the hip joint in sero-positive rheumatoid arthritis. Acta Orthop Scand 40: 346-64, 1969.

13. Lehtimaki MY, Kaarela K, Hamalainen MM: Incidence of hip involvement and need for total hip replacement in rheumatoid arthritis. An eight-year follow-up study. Scand J Rheumatol 15: 387-91, 1986.

14. Eberhardt K, Fex E, Johnsson K, Geborek P: Hip involvement in early rheumatoid arthritis. Ann Rheum Dis 54: 45-8, 1995.

15. Roberts WN, Daltroy LH, Anderson RJ: Stability of normal joint findings in persistent classic rheumatoid arthritis. Arthritis Rheum 31: 267-71, 1988

16. Lehtimaki MY, Kautiainen H, Hamalainen MM, et al: Hip involvement in seropositive rheumatoid arthritis. Survivorship analysis with a 15-year follow-up. Scand J Rheumatol 27: 406-9, 1998.

17. Hastings DE, Parker SM: Protrusio acetabuli in rheumatoid arthritis. Clin Orthop 108: 76-83, 1975.

18. Kuper HH, Van Leeuwen MA, Van Riel PL, et al: Radiographic damage in large joints in early rheumatoid arthritis: relationship with radiographic damage in hands and feet, disease activity, and physical disability. Br J Rheumatol 36: 855-60, 1997.

19. Fleming A, Benn RT, Corbett M, Wood PH: Early rheumatoid disease. II. Patterns of joint involvement. Ann Rheum Dis 35: 361-4, 1976.

20. Tanaka H, Kido K, Wakisaka A, Mine T, Kawai S: Trochanteric bursitis in rheumatoid arthritis. J Rheumatol 29: 1340-1, 2002.

21. Manaster BJ: From the RSNA Refresher Courses. Radiological Society of North America. Adult chronic hip pain: radiographic evaluation. Radiographics 20 Spec No: S3-S25, 2000.

22. Van Vugt RM, Sijbrandij ES, Bijlsma JW: Magnetic resonance imaging of the femoral head to detect avascular necrosis in active rheumatoid arthritis treated with methylprednisolone pulse therapy. Scand J Rheumatol 25: 74-6, 1996.

23. Zabinski SJ, Sculco TP, Dicarlo EF, Rivelis M: Osteonecrosis in the rheumatoid femoral head. J Rheumatol 25: 1674-80, 1998.

24. Weiss MM: Corticosteroids in rheumatoid arthritis. Semin Arthritis Rheum 19: 9-21, 1989

25. Bird PA, Oakley SP, Shnier R, Kirkham BW: Prospective evaluation of magnetic resonance imaging and physical examination findings in patients with greater trochanteric pain syndrome. Arthritis Rheum 44: 2138-45, 2001

26. Kingzett-Taylor A, Tirman PF, Feller J, et al: Tendinosis and tears of gluteus medius and minimus muscles as a cause of hip pain: MR imaging findings. AJR Am J Roentgenol 173: 1123-6, 1999.

27. Pfirmann CW, Chung CB, Theumann NH, Trudell DJ, Resnick D: Greater trochanter of the hip: attachment of the abductor mechanism and a complex of three bursae - MR imaging and MR bursography in cadavers and MR imaging in asymptomatic volunteers. Radiology 221: 469-77, 2001

28. Traycoff RB: "Pseudotrochanteric bursitis": the differential diagnosis of lateral hip pain. J Rheumatol 18: 1810-2, 1991

29. Arnett FC, Edworthy SM, Bloch DA, et al: The American Rheumatism Association 1987 revised criteria for the classification of rheumatoid arthritis. Arthritis Rheum 31: 315-24, 1988.
30. Kainberger F, Trattnig S, Czerny C, Seidl G, Kritz H, Imhof H: MRI in assessment of the systemic manifestations of rheumatological disease. Br J Rheumatol 35 Suppl 3: 40-4, 1996.

31. El-Miedany YM, Housny IH, Mansour HM, Mourad HG, Mehanna AM, Megeed MA: Ultrasound versus MRI in the evaluation of juvenile idiopathic arthritis of the knee. Joint Bone Spine 68: 222-30, 2001.

32. Larsen A, Dale K, Eek M: Radiographic evaluation of rheumatoid arthritis and related conditions by standard reference films. Acta Radiol Diagn (Stockh) 18: 481-91, 1977.

33. Sutton D: Textbook of radiology and medical imaging. London: Churchill Livingstone, 1992.

34. Resnick D, Trudell D, Fix CF: Bone and joint imaging. 2nd ed. Philadelphia: W.B. Saunders, 1996.

35. Cho KH, Park BH, Yeon KM: Ultrasound of the adult hip. Semin Ultrasound CT MR 21: 214-30, 2000.

36. Fedrizzi MS, Ronchezel MV, Hilario MO, et al: Ultrasonography in the early diagnosis of hip joint involvement in juvenile rheumatoid arthritis. J Rheumatol 24: 1820-5, 1997.

37. Murray JG, Ridley NT, Mitchell N, Rooney M: Juvenile chronic arthritis of the hip: value of contrast-enhanced MR imaging. Clin Radiol 51: 99-102, 1996

38. Walther M, Harms H, Krenn V, Radke S, Kirschner S, Gohlke F: Synovial tissue of the hip at power Doppler US: correlation between vascularity and power Doppler US signal. Radiology 225: 225-31, 2002.

39. Lamer S, Sebag GH: MRI and ultrasound in children with juvenile chronic arthritis. Eur J Radiol 33: 85-93, 2000.

40. Conway WF, Totty WG, McEnery KW: CT and MR imaging of the hip. Radiology 198: 297-307, 1996.

41. Resnick D: Diagnosis of bone and joint disorders, 4th ed, Philadelphia, Saunders, 2002

42. Furtado RNV, Ciconelli RM, Vilela SV, Fernandes ARC: Comprometimento da coluna cervical nas artropatias inflamatórias. Rev Bras Reumatol 39: 291-8, 1999.

43. Lang P, Genant HK, Jergesen HE, Murray WR: Imaging of the hip joint. Computed tomography versus magnetic resonance imaging. Clin Orthop 274: 135-53, 1992.

44. Tamisier JN, Regent D, Pere P, Thomas P, Capesius P, Gaucher A: The contribution of computerized tomography to synovial pathology. Rev Rhum Mal Osteoartic 53: 69-75, 1986.

45. Goldbach-Mansky R, Woodburn J, Yao L, Lipsky PE: Magnetic resonance imaging in the evaluation of bone damage in rheumatoid arthritis: a more precise image or just a more expensive one? Arthritis Rheum 48: 585-9, 2003.

46. Cervilla VC: Resonancia Magnética en Reumatologia. Reumatologia 13: 137-46, 1997

47. Abreu Júnior L, Vilela SA, Natour J, Fernandes ARC: Ressonância magnética (RM): contribuição para o diagnóstico e avaliação do acometimento da coluna cervical pela artrite reumatóide. Rev Bras Reumatol 35: 282-4, 1995.

48. Sadro C: Current concepts in magnetic resonance imaging of the adult hip and pelvis. Semin Roentgenol 35: 231-48, 2000.

49. Sella EMC, Fernandes ARC, Ciconelli RC, Sato EI: Ressonância Magnética nas artropatias inflamatórias. Rev Bras Reumatol 28: 269-74, 1998.

50. Oliver C, Speake S, Watt I, Dieppe P, Ratcliffe G: Advantages of an increased dose of MRI contrast agent for enhancing inflammatory synovium. Clin Radiol 51: 487-93, 1996. 
51. Creamer P, Keen M, Zananiri F, et al: Quantitative magnetic resonance imaging of the knee: a method of measuring response to intra-articular treatments. Ann Rheum Dis 56: 378-81, 1997.

52. Resnick D, Kang H. Internal Derangements of Joints-Emphasis on MR Imagins. Philadelphia, W.B. Saunders Company, 1997.

53. Goupille P, Roulot B, Akoka S, et al: Magnetic resonance imaging: a valuable method for the detection of synovial inflammation in rheumatoid arthritis. J Rheumatol 28: 35-40, 2001.

54. McGonagle D, Conaghan PG, Wakefield R, Emery P. Imaging the joints in early rheumatoid arthritis. Best Pract Res Clin Rheumatol 15: 91-104, 2001.

55. McQueen FM, Benton N, Perry D, et al: Bone edema scored on magnetic resonance imaging scans of the dominant carpus at presentation predicts radiographic joint damage of the hands and feet six years later in patients with rheumatoid arthritis. Arthritis Rheum 48: 1814-27, 2003.

56. Petersilge CA: From the RSNA Refresher Courses. Radiological Society of North America. Chronic adult hip pain: MR arthrography of the hip. Radiographics 20 Spec No: S43-52, 2000.

57. Steinbach LS, Palmer WE, Schweitzer ME: Special focus session. MR arthrography. Radiographics 22: 1223-46, 2002

58. Yamato M, Tamai K, Yamaguchi T, Ohno W: MRI of the knee in rheumatoid arthritis: Gd-DTPA perfusion dynamics. J Comput Assist Tomogr 17: 781-5, 1993.

59. Whitten CG, Moore TE, Yuh WT, Kathol MH, Renfrew DL, Walker CW: The use of intravenous gadopentetate dimeglumine in magnetic resonance imaging of synovial lesions. Skeletal Radiol 21: 215-8, 1992.

60. Ostergaard M, Klarlund M: Importance of timing of post-contrast MRI in rheumatoid arthritis: what happens during the first 60 minutes after IV gadolinium-DTPA? Ann Rheum Dis 60: 1050-4, 2001.

61. Konig H, Sieper J, Wolf KJ: Rheumatoid arthritis: evaluation of hypervascular and fibrous pannus with dynamic MR imaging enhanced with Gd-DTPA. Radiology 176: 473-7, 1990.
62. Ostergaard M, Stoltenberg M, Henriksen O, Lorenzen I: The accuracy of MRI-determined synovial membrane and joint effusion volumes in arthritis. A comparison of pre- and post-aspiration volumes. Scand J Rheumatol 24: 305-11, 1995.

63. Huang J, Stewart N, Crabbe J, et al: A 1-year follow-up study of dynamic magnetic resonance imaging in early rheumatoid arthritis reveals synovitis to be increased in shared epitope-positive patients and predictive of erosions at 1 year. Rheumatology (Oxford) 39: 407-16, 2000.

64. Waterton JC, Rajanayagam V, Ross BD, Brown D, Whittemore A, Johnstone D: Magnetic resonance methods for measurement of disease progression in rheumatoid arthritis. Magn Reson Imaging 11: 1033-8, 1993.

65. Ostergaard M, Gideon P, Henriksen O, Lorenzen I: Synovial volume - a marker of disease severity in rheumatoid arthritis? Quantification by MRI. Scand J Rheumatol 23: 197-202, 1994.

66. Ostergaard M, Hansen M, Stoltenberg M, et al: Magnetic resonance imaging-determined synovial membrane volume as a marker of disease activity and a predictor of progressive joint destruction in the wrists of patients with rheumatoid arthritis. Arthritis Rheum 42: 918-29, 1999.

67. Ostergaard M, Lorenzen I, Henriksen O: Dynamic gadoliniumenhanced MR imaging in active and inactive immunoinflammatory gonarthritis. Acta Radiol 35: 275-81, 1994.

68. Huang J, Stewart N, Crabbe J, et al: A 1-year follow-up study of dynamic magnetic resonance imaging in early rheumatoid arthritis reveals synovitis to be increased in shared epitope-positive patients and predictive of erosions at 1 year. Rheumatology (Oxford) 39: 407-16, 2000.

69. Conaghan P, Edmonds J, Emery P, et al: Magnetic resonance imaging in rheumatoid arthritis: summary of OMERACT activities, current status, and plans. J Rheumatol 28: 1158-62, 2001.

70. Ostergaard M, Klarlund M, Lassere M, et al: Interreader agreement in the assessment of magnetic resonance images of rheumatoid arthritis wrist and finger joints - an international multicenter study. J Rheumatol 28: 1143-50, 2001. 